\title{
Review of emerging therapy in refractory GERD- an evidence based approach
}

\begin{abstract}
Introduction: Proton Pump Inhibitors (PPI) has been widely used over the last 2 decades as the most preferred therapy for all GERD cases, even though it has been established that $30-40 \%$ of GERD cases do not respond to PPI's because they have visceral hyperalgesia more than hyperacidity. Newer therapies to treat patients with Refractory GERD or NERD cases can go a long way in improving the Quality of life (QOL) of these patients. This review summarizes a new approach in managing GERD by focusing on esophageal mucosal defense \& use of mucosal protective agent on NERD or refractory GERD cases. A combination of hyaluronic acid along with chondroitin sulfate dispersed in a bio adhesive carrier (Poloxamer 407) to form a macromolecular complex, which helps coat the esophageal mucosa and acts as a buffering agent to form a mechanical barrier against the reflux. The hyaluronic acid also promotes wound repair and regeneration while chondroitin sulphate has antiinflammatory and mucosal protective activity. Supportive trials have confirmed their efficacy either as adjuvant with PPIs or standalone medication to treat GERD cases including NERD and refractory GERD cases.
\end{abstract}

Keywords: GERD (gastroesophageal reflux disease), NERD (non-erosive reflux disease), PPIs (proton pump inhibitors), H2RAs (histamine2 receptor antagonists), HA (hyaluronic acid), CS (chondroitin sulfate)
Volume 9 Issue 4 - 2018

\author{
Kavi Ramnani, Sayantani Mukherjee, Dana \\ Bisharah
}

Synergy Pharma, UAE

Correspondence: Kavi Ramnani, Business Development Manager,Address: Synergy Pharma, Office 2502, 25th Floor, Business Bay, Dubai, UAE,Tel +97I50 749988I,

Email ramnanikavi@yahoo.com, kavi@synergypharma.ae

Received: May 19, 2018 | Published: August 29, 2018
Abbreviations: GERD, gastroesophageal reflux disease; NERD, non-erosive reflux disease; PPIs, proton pump inhibitors; H2RAs, histamine2 receptor antagonists; HA, hyaluronic acid; CS, chondroitin sulfate

\section{Introduction}

GERD as per Montreal classification is a long term condition where acid and bile from stomach comes into the esophagus and cause "heartburn and or regurgitation". ${ }^{1}$ The impact of GERD on loss of productivity is similar to that of other chronic diseases such as back pain and headache. ${ }^{2}$ GERD prevalence has been established in different studies(defined as onset of typical or atypical symptoms with minimum one episode in 7days) specifically in about $23.0 \%$ in South America, $18.1-27.8 \%$ in North America, 8.8-25.9\% in Europe, $11.6 \%$ in Australia, $8.7-33.1 \%$ in the Middle East and2.5-7.8\% in East Asia. ${ }^{3}$ Besides symptoms like heartburn, regurgitation and difficulty swallowing are common GERD symptoms. GERD also includes subgroups of diagnosis like: non-erosive esophageal reflux disease (NERD) and the additional pathologies results in GERD advancements, including esophageal ulcer, esophageal stricture, Barrett's esophagus, and Barrett's carcinoma (esophagus adenocarcinoma). ${ }^{1}$

\section{Treatment modalities for refractory GERD}

Discovery of $\mathrm{H} 2$ antagonists made remarkable change in GERD treatment since 1970.However, Proton pump inhibitors (PPIs), the most powerful compounds for the suppression of gastric acid secretion, have replaced $\mathrm{H} 2$ antagonists (H2RAs) in the modern times. ${ }^{4}$ In the past decade clinicians have understood that less than $30 \%$ reported GERD cases are actually suffering from Erosive reflux disease. Whereas, majority of the patient falls under NERD phenotype. Hence,
$40 \%$ of typical symptoms do not respond to PPIs because for them visceral hyperalgesia is more common than hyper acidity. ${ }^{4}$ Hence, current drugs used for treating GERD are not able to manage atypical case and hypersensitive mucosa and are not focused on mucosal defense of esophageal epithelial layer by promoting mucosal healing.

\section{Mucosal resistance- neglected parameter in GERD}

Since GERD is associated with multifactorial conditions including digestive motility, LES pressure, still composition of reflux material and esophageal mucosal resistance play very crucial role in this. ${ }^{5}$ The integrity of the esophageal mucosa in patients with a negative endoscopy was recently questioned by demonstration, using electronic microscopy, and subsequently also optical microscopy, of a new histopathological lesion represented by the dilation of the intercellular spaces. ${ }^{6,7}$ especially in biopsies performed along or $2 \mathrm{~cm}$ above the Z-line. This histological marker (Dilated intercellular space, hyperplasia of epithelium, elongation of papillae) is present in over $80 \%$ of patients with the non-erosive form of GERD, known as NERD (non-erosive reflux disease), a common finding in clinical practice $(60-70 \%$ of cases) and explains very well why these patients complain of heartburn with an intensity equal to that experienced by patients with erosive esophagitis. ${ }^{8}$ Indeed, the hydrogen ions are able to penetrate through the dilated intracellular junctions and stimulate the intramucosal nerve fibers, causing the onset of the symptoms. ${ }^{10}$ Moreover, dilated intercellular space is the outcome of damage in esophageal barrier and establish the thought that the 3 forms of GERD (NERD, ERD and Barrett's esophagus) share a common histopathologic feature within esophageal (squamous) epithelium and a common pathogenesis. ${ }^{6,11,12}$ 


\section{What role does dilation of intercellular spaces have in GERD?}

Injury to the esophageal epithelium by gastric contents causes an increase in para cellular permeability and a morphologic lesion known as Dilated Intercellular Spaces (DIS). DIS appears to play a relevant role in heartburn through contact of refluxed gastric acid with nociceptors within the esophageal mucosa and transmission of this peripheral signal to the central nervous system for cognition. In addition, DIS of the esophageal epithelium constitutes a useful and sensitive ultrastructural marker of mucosal breakage in patients with GERD nonetheless of presence of abnormal acid exposure or classical histological changes in existence of mucosal erosions (esophagitis). In fact, DIS was found in $64.8 \%$ of cases of NERD, without mucosal breaks on endoscopy, and noticeably correlated with microscopic mucosal erosions. This result strengthened the diagnostic utility of DIS in NERD. ${ }^{10}$

\section{An emerging therapy in the management of refractory GERD}

The development and adoption of drugs other than acid suppression, which are acting on different path physiological mechanisms, have been vouched in recent years. Precisely, significant attention has been paid to the use of pharmacological and physical agents aimed at potentiating the resistance related properties of esophageal mucosa, a therapeutic target, which have been underestimated till now. In same trends, a new therapy emerged in recent years and it is developed as an innovative Class III medical device. Which is a combination of hyaluronic acid along with chondroitin sulfate? These two substances are dispersed in a bio adhesive carrier (Poloxamer 407) to form a macromolecular complex, coating the esophageal mucosa and acting as a buffering agent to form a mechanical barrier against the noxious components of reflux ate, including both acidic \& non acidic reflux with prolonged action on esophageal mucosa. ${ }^{13-15}$

The class III medical device coats the esophagus and creates a mechanical barrier. Thus, reflux material is unable to damage the mucosa further. In vitro study using swine model were performed \&established the prevention ability to increase in permeability induced by acid or bile when infused with the experimental solution..$^{14,16,17}$

To establish the same result, 2 prospective placebo-controlled studies have been conducted in human model to prove the significant symptomatic relief in both group of patients with erosive reflux disease ${ }^{15}$ and non-erosive reflux disease ${ }^{16}$ cases. Recently, another prospective, double- blind, placebo-controlled, multi-centric trial was conducted in several hospitals in Italy. The main objective of the study was to confirm the effectiveness and safety profile of this medical device in combination with PPIs versus acid suppression alone in patient presented with positive NERD cases with Endoscopy negative reflux disease ${ }^{16}$ The results have found that $\mathrm{HA}+\mathrm{CS}$ combination along with PPI was able to relieve the symptoms with significant improvement in quality of life in comparison to administering PPI therapy alone. ${ }^{18}$ In the nutshell, this study demonstrated that when NERD patients were given a combination of medication (mucosal protective agent + PPI), the outcome was much better in terms of relieving severity of the symptoms like heartburn \& regurgitation when compared to only PPI therapy. Moreover almost $92 \%$ of the patient gave positive feedback on palatability \& safety of the treatment, irrespective of the time of the dosage. ${ }^{9}$

\section{Conclusion}

The association of gastric acid in this pathogenesis of GERD has been known for over 2 decades. The disease cascade algorithm unfortunately ignores the importance of mucosal resistance in the GERD evolution. The development of new mucosal protective agent, such as hyaluronic acid with chondroitin sulfate, which actually strengthen the mucosal integrity, has opened new scope of research in this particular field. Combination of this mucosal protective agent along with PPIs can help those patients in particular who are nonresponsive or partial responsive to PPI treatment. ${ }^{4}$ Lastly, it can be concluded that mucosal protective agents can extend the remission \& delay the relapse, by fulfilling the ultimate goal of GERD treatment. However, infuture large \& well designed clinical trials will be necessary to prove the above theory.

\section{Acknowledgements}

None.

\section{Conflict of interest}

The author declares no conflict of interest.

\section{References}

1. Vakil, N, van Zanten SV, Kahrilas p, et al. The Montreal definition and classification of gastroesophageal reflux disease: a global evidence-based consensus. Am J Gastroenterol. 2006;101(8):1900-1920

2. Gisbert JP, Cooper A, Karagiannis D, et al. Impact of gastroesophageal reflux disease on work absenteeism, presenteeism and productivity in daily life: A European observational study. Health Quality Life Outcomes. 2009;2009:7-90.

3. Boeckxstaens G, El-Serag HB, Smout AJ, et al. Symptomatic reflux disease: The present, the past and the future. Gut. 2014;63(7):1185-1193.

4. Edoardo Savarino, Zentilin P, Marabotto E, et al. Drugs for esophageal mucosal defense. Annals Gastroenterology. 2017;30(6):1-7.

5. De Giorgi F, Palmiero M, Esposito I, et al. Pathophysiology of gastroesophageal reflux disease. Acta Otorhinolaryngol Ital. 2006;26(5):241246.

6. Orlando RC. The integrity of the esophageal mucosa. Balance between offensive and defensive mechanisms. Best Practices Res Clin Gastroenterol. 2010;24(6):873-882.

7. Orlando LA, Orlando RC. Dilated intercellular spaces as a marker of GERD. Curr Gastroenterol Rep. 2009;11(3):190-194.

8. Zentilin P, Savarino V, Mastracci L, et al. Reassessment of the diagnostic value of histology in patients with GERD, using multiple biopsy sites and an appropriate control group. Am J Gastroenterol. 2005;100(10):22992306 .

9. Edoardo Savarino, Zentilin P, Marabotto E, et al. A review of pharmacotherapy for treating gastro-esophageal reflux disease (GERD). Expert Opinion on Pharmacotherapy. 2007;18(13):1333-1343..

10. Arul P, Phansalkar M, Alexander T, et al. Endoscope versus microscope in the diagnosis of esophageal non-erosive reflux disease: A studyof 71 cases. Malays J Pathol. 2014;36(3):181-188.

11. Orlando LA, Orlando RC. Dilated intercellular spaces as a marker of GERD. Current Gastroenterol Rep. 2009;11(3):190-194.

12. Patrick L. Gastroesophageal reflux disease (GERD): A review of conventional and alternative treatments. Altern Med Rev. 2011;16(2):11633. 
13. Scarpignato $\mathrm{C}$. Is there an effective medical therapy for extra-digestive GERD: facts and fictions. Proceeding of the conference "Extra-digestive GERD: what's next?” Parma: GASTRO; 2005. p. 16-18.

14. Di Simone MP, Baldi F, Vasina V, et al. Barrier effect of $\operatorname{ESOXX((r))~on~}$ esophageal mucosal damage: experimental study on ex-vivo swinemodel. Clin Exp Gastroenterology. 2012;5:103.

15. Palmieri B, Merighi A, Corbascio D, et al. Fixed combination of hyaluronic acid and chondroitin-sulphate oral formulation in a randomizeddouble blind, placebo controlled study for the treatment of symptoms in patients with non-erosivegastroesophageal reflux. Eur Rev Med Pharmacol Sci. 2013;17(24):3272-3278.
16. Tang M, Dettmar P, Batchelor H. Bio adhesiveesophageal bandages:protection against acid and pepsin injury. Int $J$ Pharm. 2005;292(1-2):169-177.

17. Palmieri B, Corbascio D, Capone S, et al. Preliminary clinical experience with a new natural compound in the treatment of esophagitis and gastritis:symptomatic effect. Trends Med. 2009;9(4):219-225.

18. Savarino V, Pace F, Scarpignato C. Esoxx Study Group. Randomized clinical trial: mucosal protection combined with acid suppression in the treatment of non-erosive reflux disease - efficacy of Esoxx, a hyaluronic acid-chondroitin sulphate based bio adhesive formulation. Aliment Pharmacol Ther. 2017;45(5):631-642. 